Check for updates

Cite this: RSC Adv., 2018, 8, 8739

Received 22nd December 2017 Accepted 21st February 2018

DOI: 10.1039/c7ra13572f

rsc.li/rsc-advances

\section{A p-type multi-wall carbon nanotube/Te nanorod composite with enhanced thermoelectric performance}

\begin{abstract}
Dabin Park, Hyun Ju, Taeseob Oh and Jooheon Kim (DD*
In this study, multi-walled carbon nanotube (MWCNT)/tellurium (Te) nanorod composites with various MWCNT contents are prepared and their thermoelectric properties are investigated. The composite samples are prepared by mixing Te nanorods with surface-treated MWCNTs. Te nanorods are synthesized by solution phase mixing using polyvinylpyrrolidone (PVP). The MWCNTs used in this study are surface-treated with a solution consisting of $\mathrm{H}_{2} \mathrm{SO}_{4}$ and $\mathrm{HNO}_{3}$. With increasing MWCNT content, the composite samples exhibit a reduction in the Seebeck coefficient and enhanced electrical conductivity. The maximum power factor of $5.53 \mu \mathrm{W} \mathrm{m} \mathrm{K} \mathrm{m}^{-2}$ is observed at $2 \% \mathrm{MWCNT}$ at room temperature. The thermal conductivity of the composite reduced after the introduction of MWCNTs into the Te nanorod matrix; this is attributed to the generation of heterostructured interfaces between MWCNTs and the Te nanorods. At room temperature, the composites containing 2\% MWCNTs exhibited the maximum thermoelectric figure of merit $(Z T)$, which is $\sim 3.91$ times larger than that of pure Te nanorods.
\end{abstract}

\section{Introduction}

Carbon-based fossil fuels are gradually being depleted; hence, alternative sources of energy have attracted significant interest. Some renewable energy conversion devices, such as piezoelectric, thermoelectric, fuel cells and solar cells have already been developed. Among these, thermoelectric (TE) heating and cooling devices, which are able to directly convert thermal energy into electrical energy or vice versa, are quite effective and exhibit potential for energy harvesting applications. The efficiency of TE materials is determined by a dimensionless number called the figure of merit $(Z T)$, which can be expressed as $Z T=S^{2} \sigma T / \kappa$, where $S, \sigma, \kappa$, and $T$ are the Seebeck coefficient, electrical conductivity, thermal conductivity, and absolute temperature, respectively. Theoretically, to obtain a high $Z T$ value, TE materials must have a high electrical power factor (PF $=S^{2} \sigma$ ) and low thermal conductivity.

One of the simplest ways to achieve a high PF is by using materials with high Seebeck coefficients. In general, inorganic semiconductors are promising TE materials due to their intrinsically high Seebeck coefficients owing to their crystalline structures. ${ }^{\mathbf{1 , 2}}$ Many reports are available on inorganic semiconductors and their alloys with high ZT values. Nowadays, tellurium (Te) and its alloys are widely used as thermoelectric devices due to its outstanding Seebeck coefficient. ${ }^{3-6}$ Te is a ptype semiconductor with a narrow band gap (a direct band gap of approximately $0.35 \mathrm{eV}$ at room temperature ${ }^{7}$ ) and a high

School of Chemical Engineering \& Materials Science, Chung-Ang University, Seoul 06974, Republic of Korea. E-mail: jooheonkim@cau.ac.kr
Seebeck coefficient $\left(\sim 400 \mu \mathrm{V} \mathrm{K} \mathrm{K}^{-1}\right.$ at room temperature $\left.{ }^{8,9}\right)$. However, Te is not entirely suitable to achieve a high power factor because Te has a relatively low electrical conductivity $\left(\sim 10 \mathrm{~S} \mathrm{~m}^{-1}\right.$ at room temperature $\left.{ }^{10}\right)$. Furthermore, inorganic semiconductors, including Te, still face some drawbacks apart from low electrical conductivity such as mechanical/chemical instability and lack of raw materials. ${ }^{11}$

This limitation has driven many researchers to investigate ways of enhancing the electrical conductivity of Te. ${ }^{12,13}$ One of the simplest ways of enhancing the electrical conductivity of a material is to fabricate its composites with other materials. Carbon nanotubes (CNTs) offer many advantages, such as chemical stability, ${ }^{14}$ mechanical robustness,${ }^{15}$ and high electrical conductivity. ${ }^{16}$ CNTs also exhibit excellent thermoelectric properties owing to their nanoscale, low dimensional, and holey structural features. ${ }^{17}$ Recently, many reports have been published on the enhancement of thermoelectric properties of composite materials by co-synthesis with carbon-based materials. ${ }^{18-22}$ Examples include the addition of singlewalled carbon nanotubes (SWCNTs) to a $\mathrm{Bi}_{2} \mathrm{Te}_{3}$ matrix to enhance its thermoelectric properties, incorporation of graphene in $\mathrm{a}_{2} \mathrm{Te}_{3}$ nanowire matrix to enhance its electrical conductivity while reducing the thermal conductivity, ${ }^{23}$ and the addition of SWCNTs to $\mathrm{Ag}_{2} \mathrm{Te}$ to enhance its power factor. ${ }^{24}$ In the case of multi-walled carbon nanotubes (MWCNTs), Khasimsaheb et $a .^{25}$ reported an enhancement of power factor and $Z T$ of PbTe nanocubes upon the addition of MWCNT. Similarly, Kim et al. ${ }^{\mathbf{2 6}}$ synthesized MWCNT/ $\mathrm{Bi}_{2} \mathrm{Te}_{3}$ composites and analyzed their thermoelectric properties. 
A low thermal conductivity is desired for achieving a high ZT. Recently, phonon scattering was found to be advantageous for improving the performance of TE materials., ${ }^{5,27}$ Phonon scattering is increased in nanostructures, which limit the mean free path of phonons but not that of electrons; therefore, nanostructuring can reduce the lattice thermal conductivity without affecting the electrical conductivity. ${ }^{5}$ One-dimensional (1D) nanostructures experience increased lattice scattering of phonons, which leads to a low thermal conductivity. ${ }^{28}$ Therefore, the nanostructuring of materials is one way to reduce their thermal conductivity. In previous studies, it has been shown that 1D nanostructured TE materials exhibit lower thermal conductivities than their corresponding bulk structures.5,29 Yang et $a l .^{30}$ reported a reduction in the thermal conductivity of $\mathrm{Bi}_{2} \mathrm{Te}_{3}$ nanowires fabricated by solution-phase synthesis. Another study reported that composites of 1D nanostructures of inorganic materials and carbon-based materials exhibit high thermoelectric properties as observed in the case of graphene/ $\mathrm{Bi}_{2} \mathrm{Te}_{3}$ composites. ${ }^{31}$

In this study, MWCNT/Te nanorod composite were fabricated to achieve enhanced thermoelectric properties. The Te nanorods were fabricated using a Te precursor solution via a polyvinylpyrrolidone (PVP)-assisted solution phase mixing process. The MWCNTs were surface-treated with an acidic solution to improve their reactivity. After acid treatment, carboxyl groups, which can improve the reactivity of the MWCNTs, were formed on the surfaces of the nanotubes. After the reaction of each materials, MWCNT/Te nanorod composites with varying MWCNT contents were synthesized. The thermoelectric properties, such as $S, \sigma, \kappa$, power factor and $Z T$ of the composite samples were characterized as functions of the MWCNT content. We expect that the fabrication of Te nanorods on surface-treated MWCNTs affect each other and improve the thermoelectric properties of the composites.

\section{Experimental}

\subsection{Materials}

Hydrazine monohydrate $\left(\mathrm{N}_{2} \mathrm{H}_{4} \cdot \mathrm{H}_{2} \mathrm{O}, 80 \%\right.$ ), ethylene glycol (EG, $\left.\mathrm{C}_{2} \mathrm{H}_{6} \mathrm{O}_{2}, 99.5 \%\right)$, sodium hydroxide ( $\left.\mathrm{NaOH}, 98 \%\right)$, tellurium(rv) oxide $\left(\mathrm{TeO}_{2}, 99 \%\right)$, ethanol $\left(\mathrm{C}_{2} \mathrm{H}_{5} \mathrm{OH}, 94 \%\right)$, nitric acid $\left(\mathrm{HNO}_{3}\right.$, $60 \%)$, and sulfuric acid $\left(\mathrm{H}_{2} \mathrm{SO}_{4}, 95 \%\right)$ were purchased from Daejung Chemicals \& Metals Co. (Seoul, Korea), PVP (molecular weight (MW) $=\sim 40$ 000) was purchased from Sigma-Aldrich (St. Louis, USA). The multi-walled carbon nanotubes used in this study were supplied by Hanwha Nanotech (Seoul, Korea).

\subsection{Synthesis of Te nanorods}

Te nanorods were prepared by mixing $1.92 \mathrm{~g}$ of $\mathrm{TeO}_{2}(\mathrm{MW}=$ 159.6), $2.4 \mathrm{~g}$ of $\mathrm{NaOH}$ (MW $=40$ ), and $0.8 \mathrm{~g}$ of PVP in a roundbottomed flask, to which $80 \mathrm{~mL}$ of EG was subsequently added. This solution was stirred and heated to $393 \mathrm{~K}$ and was followed by the injection of $4.9 \mathrm{~mL}$ of $\mathrm{N}_{2} \mathrm{H}_{4} \cdot \mathrm{H}_{2} \mathrm{O}$ into the mixture. Due to the formation of tellurium oxide colloids, the color of the mixture turned white within a few seconds. After the injection of $\mathrm{N}_{2} \mathrm{H}_{4} \cdot \mathrm{H}_{2} \mathrm{O}$, the color of the solution turned dark gray. The reaction was allowed to continue for $90 \mathrm{~min}$, after which a dark blue solution of Te nanorods was obtained. The resulting mixture was poured into deionized (DI) water containing $10 \mathrm{vol} \%$ hydrazine monohydrate and stirred vigorously to eliminate any residual surfactants and reactants. The resulting solution was subsequently centrifuged with the addition of volumetric water twice to remove the supernatant and redispersed at least twice to increase the purity of the resulting powder. Finally, the obtained product was dried in a vacuum oven at $333 \mathrm{~K}$ for $24 \mathrm{~h}$.

\subsection{Preparation of surface-treated MWCNTs}

Pristine MWCNTs $(3.0 \mathrm{~g})$ were stirred in $800 \mathrm{~mL}$ of a mixed solution of $\mathrm{H}_{2} \mathrm{SO}_{4}$ and $\mathrm{HNO}_{3}(3: 1 \mathrm{v} / \mathrm{v}$ ratio). Subsequently, this solution was heated to $353 \mathrm{~K}$ and stirred for $6 \mathrm{~h}$. The purpose of this chemical functionalization step was to ensure that carboxyl groups were attached to the surfaces of the CNTs. The functionalized CNTs were centrifuged several times and filtered with DI water until the $\mathrm{pH}$ was neutral. They were then dried in a vacuum oven at $333 \mathrm{~K}$ for $24 \mathrm{~h}$.

\subsection{Fabrication of MWCNT/Te nanorod composites}

Initially, $0.1 \mathrm{~g}$ of the Te nanorods were suspended in $50 \mathrm{~mL}$ of ethanol containing 1, 2, 3, 5, and $10 \mathrm{wt} \%$ of MWCNTs. Subsequently, the mixtures were subjected to ultrasonication for $30 \mathrm{~min}$. Later, the mixtures were stirred at $353 \mathrm{~K}$ for $1 \mathrm{~h}$ with an appropriate amount of $\mathrm{N}_{2} \mathrm{H}_{4} \cdot \mathrm{H}_{2} \mathrm{O}$ to reduce dispersion. The resulting products were washed and filtered with DI water, and dried at $333 \mathrm{~K}$ for $24 \mathrm{~h}$. The final composites were ground into fine powders. They were then loaded into a Fe mold and pressed at $473 \mathrm{~K}$ under a pressure of $50 \mathrm{MPa}$ for $5 \mathrm{~min}$.

\subsection{Characterization}

X-ray diffraction (XRD; New D8 Advance, Bruker AXS) was used to characterize the crystalline structure of the synthesized materials. The analysis was performed at $40 \mathrm{~mA}$ and $40 \mathrm{kV}$ at a scan rate of $1^{\circ} \mathrm{s}^{-1}$, with $2 \theta$ ranging from 5 to $70^{\circ}$, using $\mathrm{Cu} \mathrm{K} \alpha$ radiation $(\lambda=0.154056 \mathrm{~nm})$. The binding energy peaks of the synthesized materials were analyzed by X-ray photoelectron spectroscopy (XPS; VG-Microtech, ESCA2000). The morphologies and structural properties of the as-prepared products were analyzed by field-emission scanning electron microscopy (FESEM, SIGMA, Carl Zeiss). The elemental composition of the samples was analyzed by energy-dispersive X-ray spectroscopy (EDS, NORAN system 7, Thermo Scientific). Fourier transform infrared (FT-IR, Bio-rad FTS-1465) spectra of the composites were obtained using pressed, disk-shaped pellets of the samples mixed with potassium bromide $(\mathrm{KBr})$, with an average of 32 scans in the $500-4000 \mathrm{~cm}^{-1}$ wavenumber range A homemade device containing a pair of voltmeters and thermocouples was used to measure the Seebeck coefficient. The value was determined from the linear relationship between the thermal electromotive force $(\Delta V)$ and the through-plane temperature difference $(\Delta T)$ of the composite films $(S=\Delta V / \Delta T)$. The electrical conductivity of the samples was measured using the 4point-probe method and the thickness of the sample was 
measured using a digital micrometer. The thermal conductivity of the samples was determined using the formula $\kappa=\alpha \rho C_{\mathrm{p}}$, where $\alpha$ is the thermal diffusivity, $\rho$ is the bulk density, and $C_{\mathrm{p}}$ is the specific heat of the materials. The thermal diffusivity of the samples was measured using a LFA 447 Nanoflash (NETZSCH) instrument. The specific heat of the samples was measured via differential scanning calorimetry (DSC; 131 evo, Setaram Instrumentation) in the temperature range of $-20^{\circ} \mathrm{C}$ to $120^{\circ} \mathrm{C}$ at a heating rate of $10 \mathrm{~K} \mathrm{~min}^{-1}$ under $\mathrm{N}_{2}$ atmosphere. All measurements were performed in the same direction to prevent anisotropy of the samples.

\section{Results and discussion}

Fig. 1 shows the overall scheme of the synthesis of MWCNT/Te nanorod composite. $\mathrm{TeO}_{2}, \mathrm{NaOH}$, and PVP were mixed with EG and heated until the temperature of the solution reached $393 \mathrm{~K}$; later, $\mathrm{N}_{2} \mathrm{H}_{4} \cdot \mathrm{H}_{2} \mathrm{O}$ was injected into the solution. PVP plays a key role as a surfactant in the synthesis of Te nanorod. Previous studies have reported that a linear polymer can react with inorganic ions to form chain-shaped intermediates. ${ }^{32,33}$ However, in this study, PVP might also serve as a directing template for the synthesis of nanorod. In other words, PVP induces 1D growth and controls the growth rate of the Te nanorods. At this stage, the nucleation of the $\mathrm{Te}^{2-}$ ions occurred and they were reduced to elemental Te to form solid crystal nuclei. As the reduction continued, the growth of Te ions to Te nanorods was accelerated.

XRD analysis was conducted to determine the crystalline phases of the synthesized Te nanorods and the results are shown in Fig. 2(a). All the peaks shown in these patterns can be indexed to the hexagonal phase of Te (JCPDS, no. 13-1452) and no other peaks could be found, indicating that only elemental Te grains with high crystallinity were obtained.

The synthesized Te nanorods were further analyzed by XPS. The XPS data corresponding to the Te nanorods is shown in Fig. 2(b) and (c). A high-resolution spectrum of the Te $3 \mathrm{~d}$ region (Fig. 2(c)) shows peaks at about $583.38 \mathrm{eV}$ and $572.98 \mathrm{eV}$, which correspond to the Te $3 \mathrm{~d}_{3 / 2}$ and $\mathrm{Te} 3 \mathrm{~d}_{5 / 2}$ binding energies of Te, respectively. In addition, the two peaks at $586.88 \mathrm{eV}$ and $576.48 \mathrm{eV}$ (Fig. 2(c)) can be assigned as Te(rv) $3 \mathrm{~d}$ binding energy, which indicates the oxidation of Te. It demonstrates that the outer surface of the Te nanorods is easily oxidized in air due to their 1D nanostructure. It can be concluded that the atoms at the surface have a lower coordination number and hence are more active than the inner atoms. Owing to this feature, the nanostructures could be more easily oxidized in air. Several previous studies reported that nanomaterials could be oxidized easily in air. ${ }^{34}$ Our XPS data is consistent with such results. ${ }^{4}$ This implies that the Te nanorods were successfully synthesized by the chosen fabrication process, which was further confirmed by FE-SEM and EDS analyses, the details of which are provided in the following sections.

The fabricated 1D Te nanorod samples are clearly visible in the FE-SEM images shown in Fig. 3(a) and (b), which show the presence of a large number of randomly distributed wire-like structures. It can be observed that the obtained products are mostly cylindrically shaped rods of relatively uniform size. Each Te nanorod is $\sim 15 \mathrm{~nm}$ in length, and $\sim 600 \mathrm{~nm}$ in diameter. FESEM analysis thus confirms that Te nanorods were successfully prepared. This result was further verified by EDS atomic mapping for Te (Fig. 3(c)). The EDS spectrum collected from a specific region (Fig. 3(c)) indicated the presence of Te. From these results, we can infer that Te nanorods were successfully synthesized.

Prior to the synthesis of MWCNT/Te composites, the surfaces of the MWCNTs were treated by $\mathrm{HNO}_{3}$ and $\mathrm{H}_{2} \mathrm{SO}_{4}$. The treatment resulted in the formation of carboxylic groups on the surface of MWCNTs while keeping the MWCNT length intact. This reaction also leads to the elimination of impurities. Additionally, the carboxyl groups on the surface enhance the reactivity of MWCNTs, so this treatment make MWCNTs with Te nanorod easy.

FT-IR was performed to analyze the chemical bonding and types of functional groups grafted onto the MWCNTs. Fig. 4 illustrates the FT-IR spectra of the MWCNTs and acid-treated

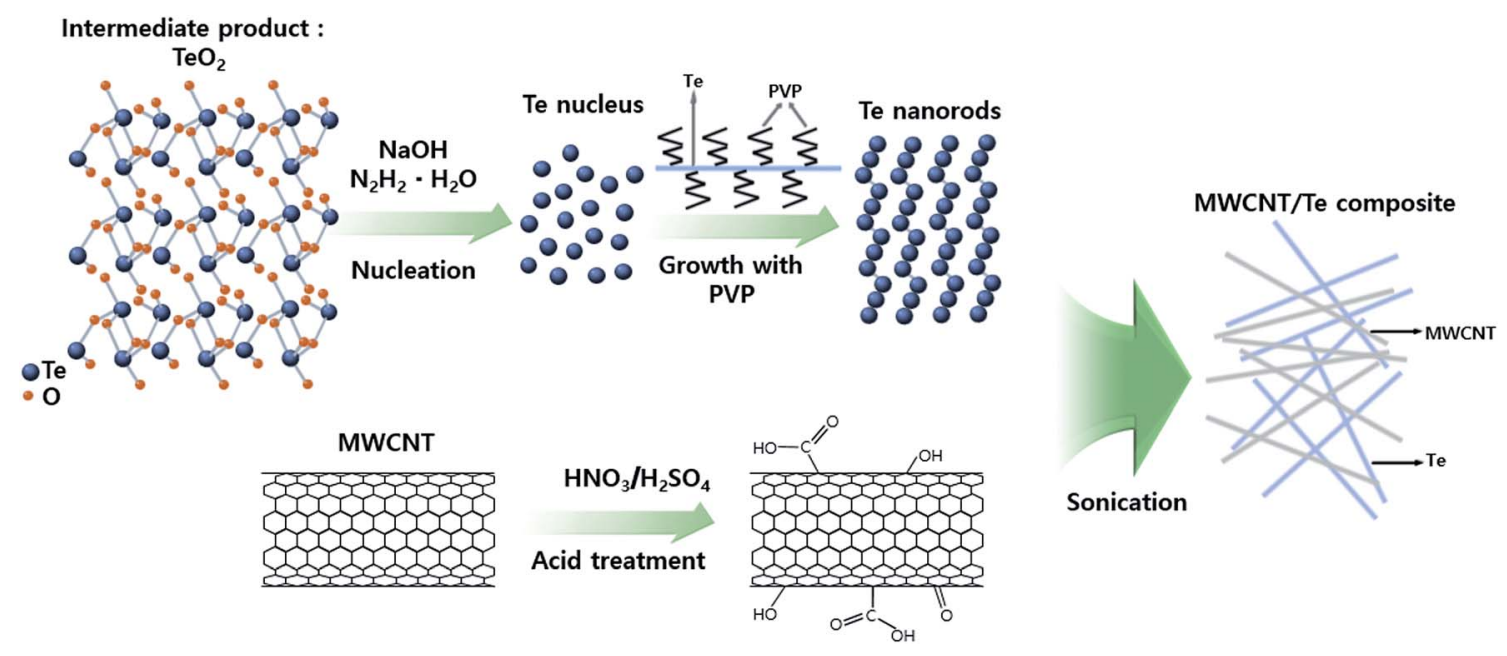

Fig. 1 Schematic illustration of the fabrication of MWCNT/Te nanorod composite. 

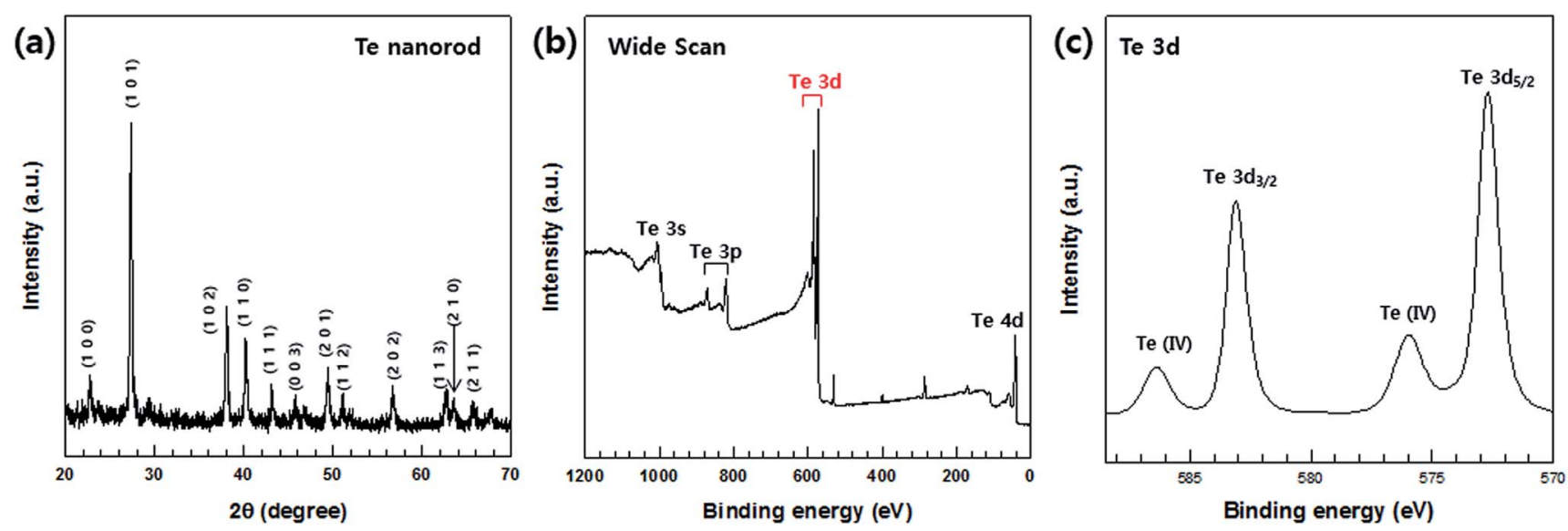

Fig. 2 (a) XRD patterns and (b and c) XPS spectra of Te nanorods. (b) Wide XPS scan for Te. (c) Te 3d spectrum.
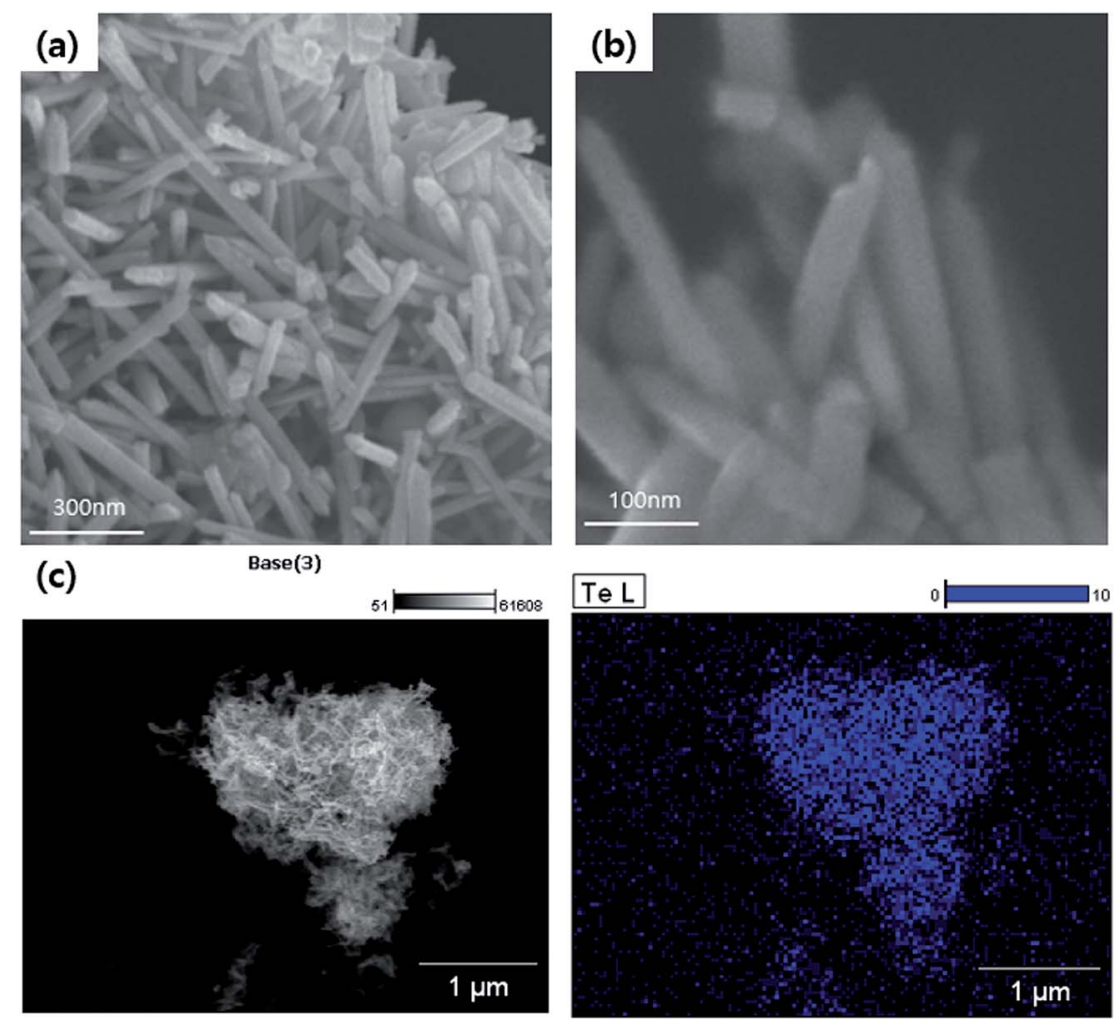

Fig. 3 (a) Low and (b) high-magnification FE-SEM images of Te nanorods. (c) Particle surface morphology and atomic mapping of Te nanorods.

MWCNTs. Although the quality of the spectra is low because of the MWCNT texture, the data substantiate the formation of carboxyl group on the surfaces of the MWCNTs. The MWCNT spectrum denotes $\mathrm{O}-\mathrm{H}$ stretching vibrations as a broad absorption band at $3400 \mathrm{~cm}^{-1}$ and as a relatively narrow peak at $1410 \mathrm{~cm}^{-1}$. The absorption peak at $1715 \mathrm{~cm}^{-1}$ corresponds to the typical stretching vibration of the $\mathrm{C}=\mathrm{O}$ groups in the carboxyl units. The characteristic peak at about $1636 \mathrm{~cm}^{-1}$ can be assigned to the vibration of the aromatic $\mathrm{C}=\mathrm{C}$ bonds. The broad peaks at $1180 \mathrm{~cm}^{-1}$ could be assigned to the $\mathrm{C}-\mathrm{O}$ stretch of the alkoxy group. ${ }^{35}$
XPS analysis was relied upon to confirm the oxidation of the MWCNTs; the wide-survey and high-resolution spectra of the MWCNTs are shown in Fig. 5(a)-(c), respectively. The C 1s region of the XPS spectrum reveals the presence of $\mathrm{C}-\mathrm{C}$ bonding in MWCNTs at a binding energy $284.6 \mathrm{eV}, \mathrm{C}-\mathrm{O}$ bonding at $287.4 \mathrm{eV}, \mathrm{C}-\mathrm{H}$ bonding at $285 \mathrm{eV}$, and $\mathrm{C}=\mathrm{O}$ bonds of the carboxylated group at $288 \mathrm{eV}$; furthermore, at a binding energy of $289 \mathrm{eV}$, carbonyls consistent with carboxylated groups were formed.

The XRD patterns of the MWCNT/Te composites with various MWCNT contents $(1,2,3,5$ and 10\%) are shown in 


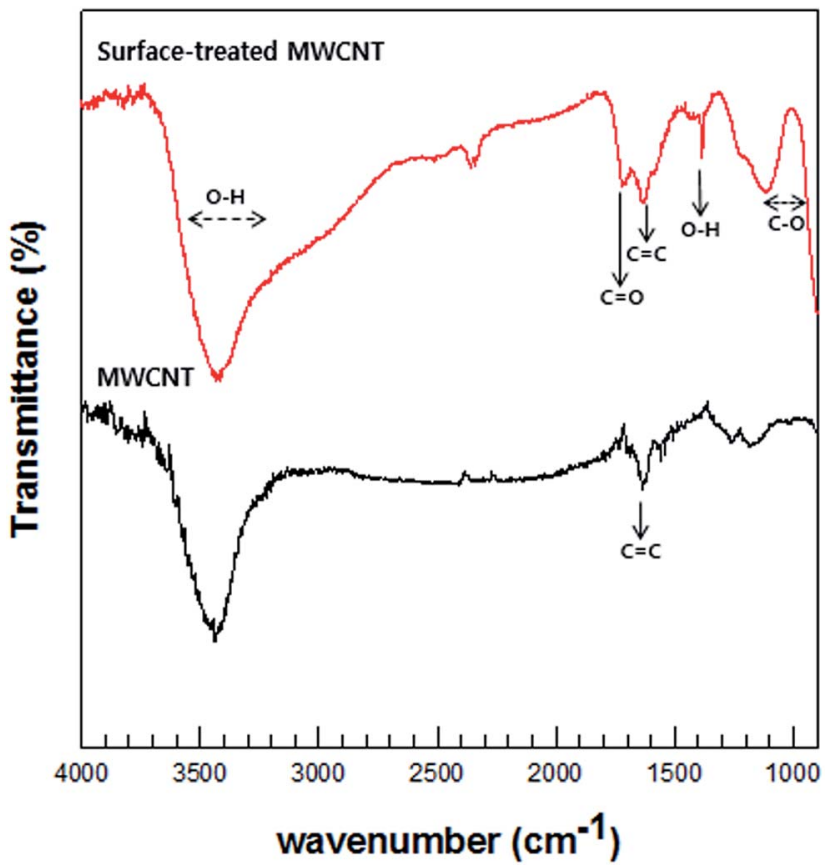

Fig. 4 Typical FT-IR spectra of pristine MWCNT and surface-treated MWCNT.

Fig. 6. The diffractograms of the composite are almost similar to that of the Te nanorods. However, with an increase in the MWCNT content, the intensities of the peaks corresponding to Te decrease in the XRD patterns of the composites.

FE-SEM analysis was carried out to observe the microscopic morphology of the fabricated MWCNT/Te nanorod composites (Fig. 7 and 8). Fig. 7 shows the low and high magnification cross-sectional FE-SEM images of the MWCNT/Te nanorod composites $2 \mathrm{wt} \%$ of MWCNT contents. These images confirm that the MWCNTs were well dispersed in the Te nanorod matrix. However, after $3 \mathrm{wt} \%$, MWCNT dispersion in Te matrice is not uniform, from $5 \mathrm{wt} \%$, MWCNT aggregates themselves. These trend is confirmed at Fig. 8.

Prior to measuring the TE characteristics, using a hot press, the MWCNT/Te nanorod composites were fabricated into disks of $12.7 \mathrm{~mm}$ diameter; pure Te disks were also prepared.

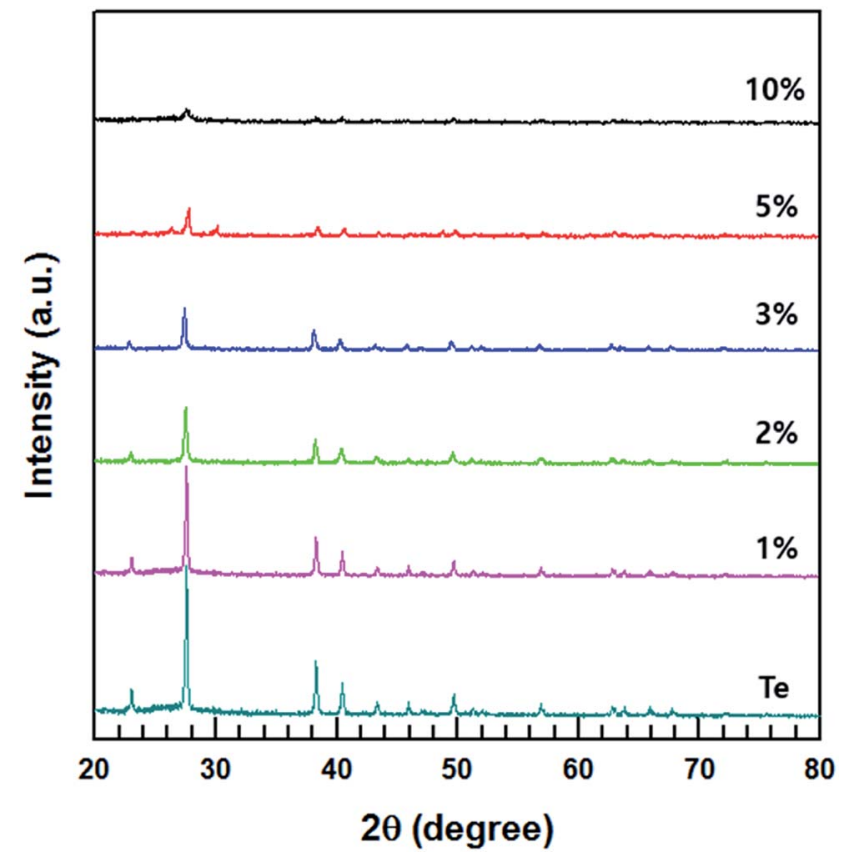

Fig. 6 XRD patterns of MWCNT/Te nanorod composites with different MWCNT contents and Te nanorods.

The electrical conductivities of the MWCNT/Te nanorod composite $(1,2,3,5$ and $10 \%)$ at room temperature are shown in Fig. 8. The electrical conductivity of the pure Te sample was measured (Fig. 9) to be $16.3 \mathrm{~S} \mathrm{~m}^{-1}$. Pristine CNTs exhibit a very high electrical conductivity, ${ }^{36}$ which is much higher than that of the pure Te sample. As shown in Fig. 9, the electrical conductivity of the composites increases with increasing MWCNT content and at $10 \mathrm{wt} \%$, the maximum value of $334.2 \mathrm{~S} \mathrm{~m}^{-1}$ was obtained. This increasing trend in electrical conductivity is attributed to the electrical conductivity of MWCNTs, which is relatively higher than that of the Te nanorods.

The Seebeck coefficients of the MWCNT/Te nanorod composites containing 1, 2, 3, 5 and 10\% MWCNT at room temperature are also shown in Fig. 9. Unlike the electrical conductivity, the Seebeck coefficients experience a decrease with an increase in the MWCNT content. The Seebeck
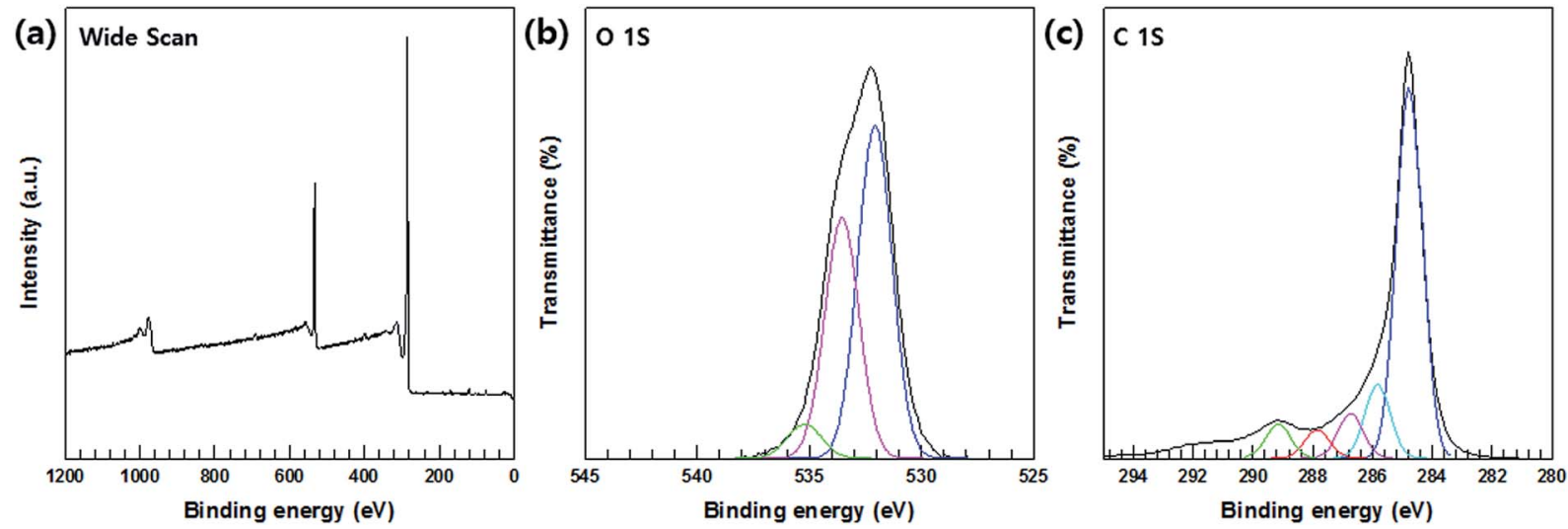

Fig. 5 XPS spectra surface-treated MWCNTs. (a) Wide XPS scan (b) $O 1$ s spectrum. (c) C 1s spectrum. 


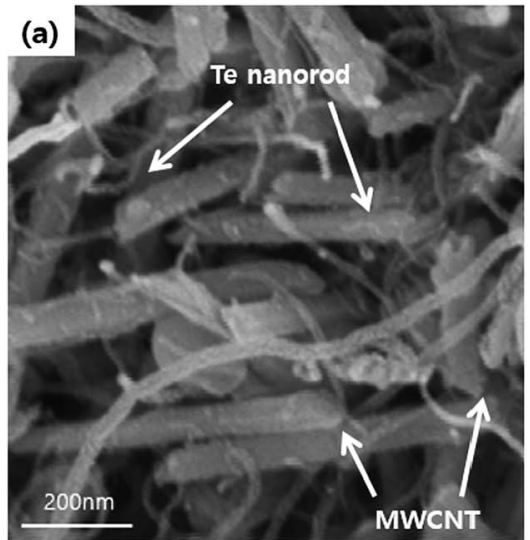

(b)

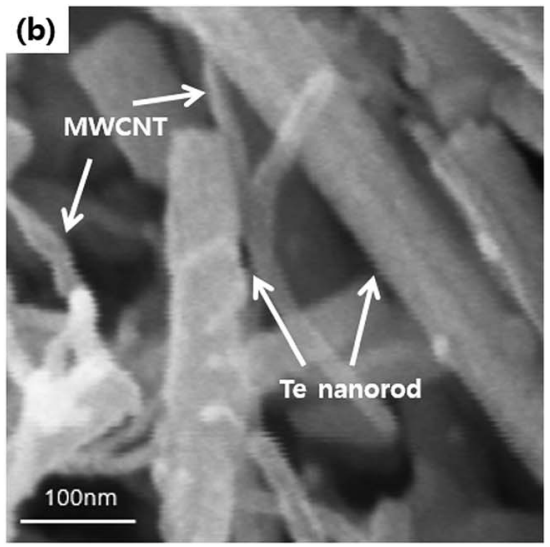

Fig. 7 (a) Low and (b) high-magnification FE-SEM images of MWCNT/Te nanorod composites with 2 wt\% MWCNT.

coefficient of the pristine Te nanorods is $404.5 \mu \mathrm{V} \mathrm{K}^{-1}$, which is similar to the value reported previously. ${ }^{3,4}$ The Seebeck coefficient of pure MWCNTs is $\sim 12.2 \mu \mathrm{V} \mathrm{K}{ }^{-1}$, which is analyzed in previous studies $;^{36}$ the Seebeck coefficient of MWCNTs is much lower than that of the Te nanorods. The decreasing trend observed in the Seebeck coefficients of the composite samples is due to the difference in the Seebeck coefficients of the two materials. In addition, the Seebeck coefficients of both MWCNTs and Te nanorod have positive values, which indicates that the two materials exhibit p-type electrical transport behavior.

\subsection{Nanorod matrix to form a composite}

Fig. 10 illustrates the dependence of the total thermal conductivity of the composites on the MWCNT content. The total thermal conductivity $(\kappa)$ consists of a lattice thermal term $\left(\kappa_{1}\right)$ corresponding to phonons and an electronic term $\left(\kappa_{\mathrm{e}}\right)$ corresponding to the charge carriers $\left(\kappa=\kappa_{1}+\kappa_{\mathrm{e}}\right) . \kappa_{\mathrm{e}}$ is estimated from the Wiedemann-Franz law $\kappa_{\mathrm{e}}=L \sigma T$ where $L$ is the Lorentz number $\left(L=2.45 \times 10^{-8} \mathrm{~W} \Omega \mathrm{K}^{-2}\right) \cdot^{37,38}$ The total thermal conductivity is mainly dependent on the lattice term $\kappa_{1}$ because of the relatively small contribution of the electronic term. Thus, the lattice scattering of phonons is the dominant factor that determines $\kappa$ for the composite. The findings in this study show that the incorporation of MWCNT particles into the Te matrices

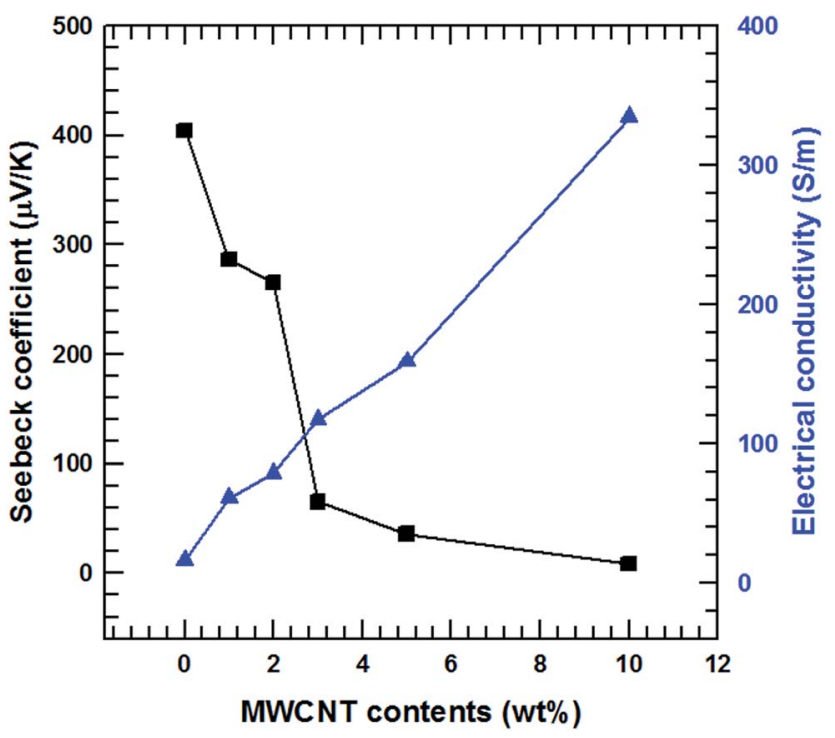

Fig. 9 Seebeck coefficient and electrical conductivity of the MWCNT/ Te nanorod composite with different MWCNT contents.

generated MWCNT/Te heterointerfaces, providing effective phonon-scattering centers. Therefore, the $\kappa_{1}$ values of the composites were lower than those of the pure Te samples. The $\kappa$ value of the pristine Te sample decreased until the MWCNT
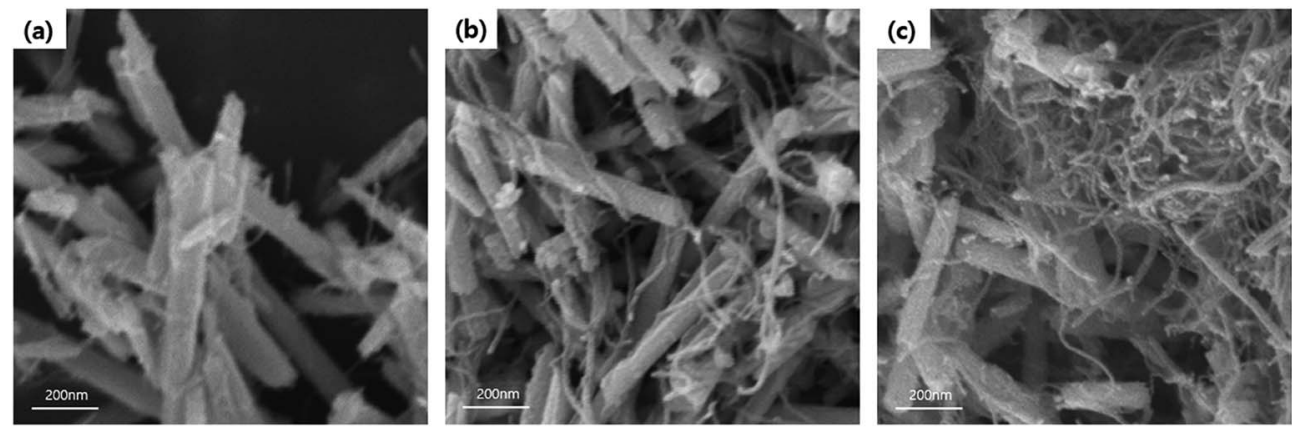

Fig. 8 FE-SEM images MWCNT/Te nanorod composites with various MWCNT contents (a) 1 wt\%, (b) 3 wt \%, and (c) 5 wt\%. 


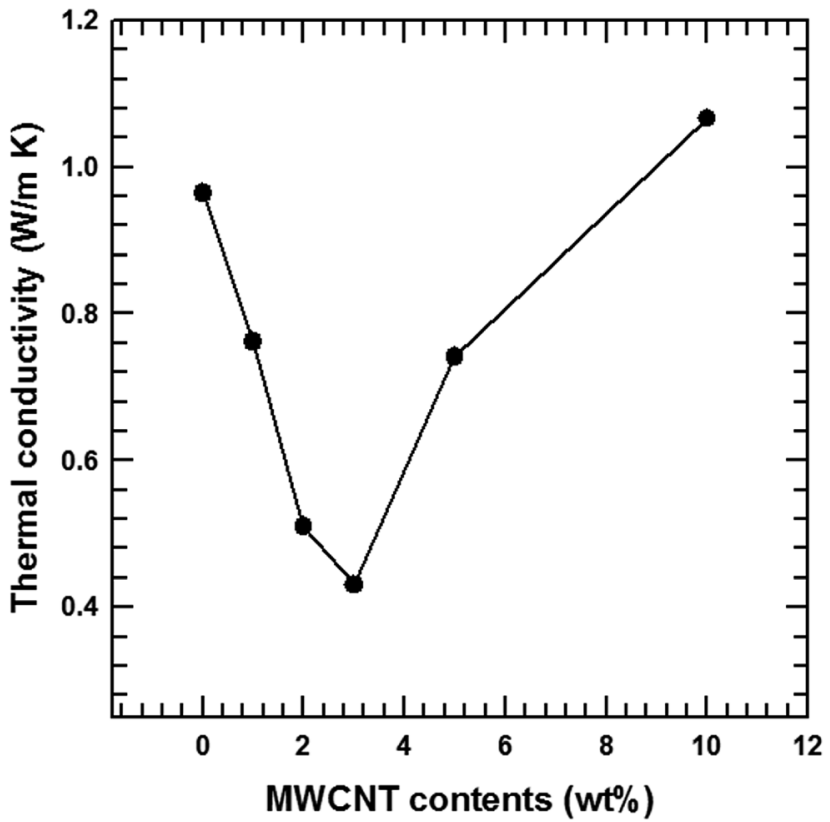

Fig. 10 Thermal conductivity of the MWCNT/Te nanorod composites with different MWCNT contents.

reached $3 \mathrm{wt} \%$ due to the phonon scattering effect. However, above $3 \mathrm{wt} \%$ of MWCNTs, the $\kappa$ values tended to increase because of the outstanding thermal conductivity of the MWCNTs. ${ }^{39,40}$ This value overwhelms the reduction in the $\kappa$ value resulting from phonon scattering.

The thermoelectric performances of the MWCNT/Te nanorod composites were evaluated by analyzing the power factor as well as the thermoelectric figure of merit $\left(Z T=S^{2} \sigma T / \kappa\right)$. The power factors of the MWCNT/Te nanorod composites with various MWCNT contents are shown in Fig. 11(a). The composite sample with $2 \%$ MWCNT shows the highest power factor $\left(5.53 \mu \mathrm{W} \mathrm{m} \mathrm{K}{ }^{-2}\right)$. This value is $\sim 2.07$ times larger than the power factor of pure Te nanorods. The addition of small amounts of MWCNTs to the composite resulted in a high power factor because of the high electrical conductivity of the
MWCNTs. However, in composite samples with MWCNT contents greater than $2 \%$, the power factor decreased due to the low Seebeck coefficient of the MWCNT.

The thermoelectric figure of merit, $Z T$, was also evaluated and the results are shown in Fig. 11(b). Due to the improved power factor and low thermal conductivity of the MWCNT/Te nanorod composites, the $Z T$ values of the composites are much higher than that of the pristine Te nanorods. At room temperature, the maximum $Z T$ value of $4.5 \times 10^{-3}$, was exhibited the composite with $2 \%$ MWCNT, which is $~ 3.91$ times larger than that of pure Te nanorods. These results indicate that the introduction of MWCNTs can synergistically enhance the thermoelectric properties of Te nanorods.

The MWCNT/Te nanorod composites studied in this investigation exhibited improved thermoelectric characteristics. The high electrical conductivity of the MWCNTs can enhance the thermoelectric properties of the composites. In addition, the thermal conductivities of the MWCNT/Te nanorods composites were found to be low, owing to the strong phonon scattering at the nanostructured interfaces between the nanorods and the MWCNTs. The results of this study suggest that the synthesis of synergetic networks consisting of Te nanorods and MWCNTs can enhance the thermoelectric properties of the MWCNT/Te composites.

\section{Conclusion}

MWCNT/Te nanorod composites with varying MWCNT contents were fabricated, and their thermoelectric properties were investigated. During the solution-phase synthesis of Te nanorods, PVP helps in the structuring of wire-like Te structure. It holds the each particles, so the particles grow with 1D nanostructure. The MWCNTs used in this study were treated with an acidic solution of $\mathrm{H}_{2} \mathrm{SO}_{4}$ and $\mathrm{HNO}_{3}$ to generate carboxylic groups on their surfaces. The MWCNTs were uniformly distributed throughout the Te nanorod matrix by ultrasonication and vacuum filtering, providing a welldispersed solution. The nanostructures and morphologies of the samples were evaluated by FE-SEM, EDS, XRD, XPS and FT-
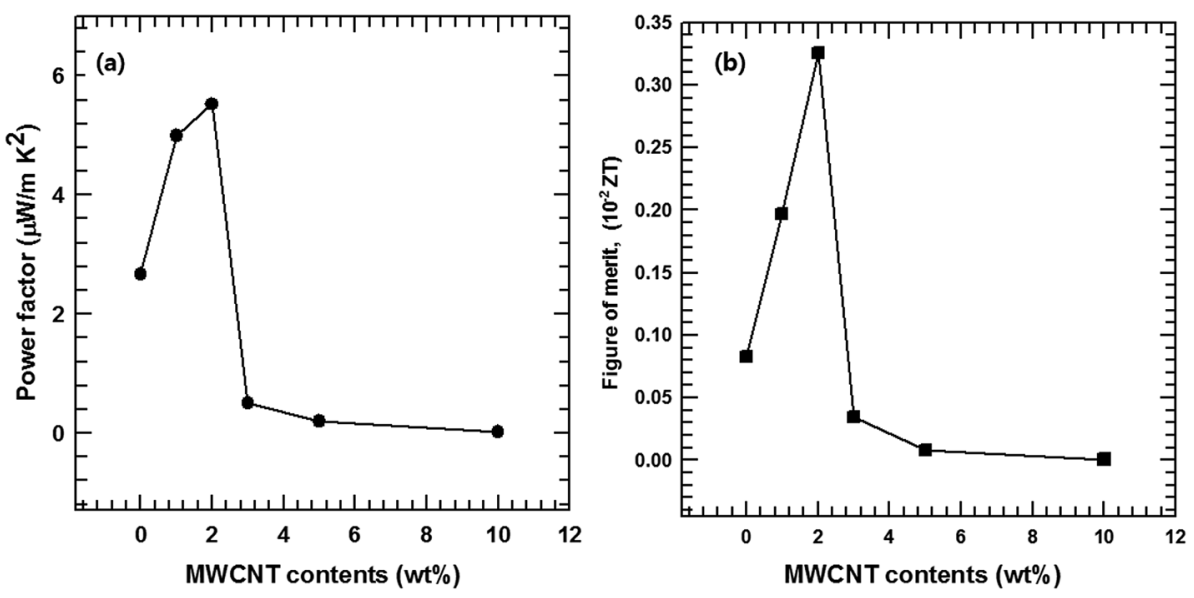

Fig. 11 (a) Power factor and (b) figure of merit (ZT) of the MWCNT/Te nanorod composite with different MWCNT contents. 
IR analyses. We also investigated the effect of composite formation on the thermoelectric properties of the Te nanorods. The Seebeck coefficient of the composite decreased with increasing MWCNT content. However, the electrical conductivity of the composites increased with an increase in the MWCNT content. As a result, composites with 2\% MWCNT exhibited the largest power factor of $5.52 \mu \mathrm{W} \mathrm{m} \mathrm{K}{ }^{-2}$ at room temperature. This value is $\sim 200 \%$ higher than that of pure Te nanorods. The reduction in the thermal conductivity of the composites is attributed to the phonon scattering effect. 1D nanostructures exhibit increased phonon scattering, as a result of which the lattice thermal conductivity is reduced. The dependence of thermal conductivity on the MWCNT content was also analyzed. Te highest figure of merit was obtained with the composite containing $2 \%$ of MWCNT. It is $~ 3.91$ times larger than that of pure Te nanorods.

\section{Conflicts of interest}

There are no conflicts to declare.

\section{Acknowledgements}

This work was supported by the NRF Grant funded by the Ministry of Education (2017R1D1A1B03029212).

\section{References}

1 W. Liu, X. Yan, G. Chen and Z. Ren, Nano Energy, 2012, 1, 4256.

2 S. K. Bux, J.-P. Fleurial and R. B. Kaner, Chem. Commun., 2010, 46, 8311-8324.

3 C. Dun, C. A. Hewitt, H. Huang, D. S. Montgomery, J. Xu and D. L. Carroll, Phys. Chem. Chem. Phys., 2015, 17, 8591-8595.

4 H. Park, W. Son, S. H. Lee, S. Kim, J. J. Lee, W. Cho, H. H. Choi and J. H. Kim, CrystEngComm, 2015, 17, 10921097.

5 B. Poudel, Q. Hao, Y. Ma, Y. Lan, A. Minnich, B. Yu, X. Yan, D. Wang, A. Muto and D. Vashaee, Science, 2008, 320, 634638.

6 G. Tai, W. Guo and Z. Zhang, Cryst. Growth Des., 2008, 8, 2906-2911.

7 F. Liang and H. Qian, Mater. Chem. Phys., 2009, 113, 523-526. 8 S. Lin, W. Li, Z. Chen, J. Shen, B. Ge and Y. Pei, Nat. Commun., 2016, 7, 10287.

9 K. C. See, J. P. Feser, C. E. Chen, A. Majumdar, J. J. Urban and R. A. Segalman, Nano Lett., 2010, 10, 4664-4667.

10 S. K. Yee, N. E. Coates, A. Majumdar, J. J. Urban and R. A. Segalman, Phys. Chem. Chem. Phys., 2013, 15, 40244032 .

11 J. R. Sootsman, D. Y. Chung and M. G. Kanatzidis, Angew. Chem., Int. Ed., 2009, 48, 8616-8639.

12 D. Park, H. Ju and J. Kim, Ceram. Int., 2017, 43, 11156-11162.

13 Y. Zhang, H. Wang, S. Kraemer, Y. Shi, F. Zhang, M. Snedaker, K. Ding, M. Moskovits, G. J. Snyder and G. D. Stucky, ACS Nano, 2011, 5, 3158-3165.
14 R. H. Baughman, A. A. Zakhidov and W. A. De Heer, Science, 2002, 297, 787-792.

15 J. N. Coleman, U. Khan, W. J. Blau and Y. K. Gun'ko, Carbon, 2006, 44, 1624-1652.

16 P. Ajayan, Chem. Rev., 1999, 99, 1787-1800.

17 I. Kunadian, R. Andrews, M. P. Mengüç and D. Qian, Carbon, 2009, 47, 589-601.

18 L. Liang, C. Gao, G. Chen and C.-Y. Guo, J. Mater. Chem. C, 2016, 4, 526-532.

19 X. Hu, G. Chen and X. Wang, Compos. Sci. Technol., 2017, 144, 43-50.

20 G. Wu, Z.-G. Zhang, Y. Li, C. Gao, X. Wang and G. Chen, ACS Nano, 2017, 11, 5746-5752.

21 G. Wu, C. Gao, G. Chen, X. Wang and H. Wang, J. Mater. Chem. A, 2016, 4, 14187-14193.

22 D. Tan, J. Zhao, C. Gao, H. Wang, G. Chen and D. Shi, ACS Appl. Mater. Interfaces, 2017, 9, 21820-21828.

23 Y. Zhang, X. Wang, W. Yeoh, R. Zheng and C. Zhang, Appl. Phys. Lett., 2012, 101, 031909.

24 W. Zhao, H. T. Tan, L. P. Tan, S. Fan, H. H. Hng, Y. C. F. Boey, I. Beloborodov and Q. Yan, ACS Appl. Mater. Interfaces, 2014, 6, 4940-4946.

25 B. Khasimsaheb, N. K. Singh, S. Bathula, B. Gahtori, D. Haranath and S. Neeleshwar, Current Applied Physics, 2017, 17, 306-313.

26 K. T. Kim, S. Y. Choi, E. H. Shin, K. S. Moon, H. Y. Koo, G.-G. Lee and G. H. Ha, Carbon, 2013, 52, 541-549.

27 A. Minnich, M. Dresselhaus, Z. Ren and G. Chen, Energy Environ. Sci., 2009, 2, 466-479.

28 M. S. Dresselhaus, G. Chen, M. Y. Tang, R. Yang, H. Lee, D. Wang, Z. Ren, J. P. Fleurial and P. Gogna, Adv. Mater., 2007, 19, 1043-1053.

29 H. Ju and J. Kim, J. Alloys Compd., 2016, 664, 639-647.

30 L. Yang, Z.-G. Chen, M. Hong, G. Han and J. Zou, ACS Appl. Mater. Interfaces, 2015, 7, 23694-23699.

31 H. Ju, M. Kim and J. Kim, Chem. Eng. J., 2015, 275, 102-112. 32 H.-S. Qian, S.-H. Yu, J.-Y. Gong, L.-B. Luo and L.-f. Fei, Langmuir, 2006, 22, 3830-3835.

33 Z. Liu, Z. Hu, J. Liang, S. Li, Y. Yang, S. Peng and Y. Qian, Langmuir, 2004, 20, 214-218.

34 A. Samal and T. Pradeep, J. Phys. Chem. C, 2009, 113, 1353913544.

35 V. Gupta and T. A. Saleh, in Carbon Nanotubes - From Research to Applications, InTech, 2011.

36 C. Meng, C. Liu and S. Fan, Adv. Mater., 2010, 22, 535-539.

37 M. T. Pettes, J. Maassen, I. Jo, M. S. Lundstrom and L. Shi, Nano Lett., 2013, 13, 5316-5322.

38 O. Bubnova and X. Crispin, Energy Environ. Sci., 2012, 5, 9345-9362.

39 A. Usenko, D. Moskovskikh, M. Gorshenkov, A. Korotitskiy, S. Kaloshkin, A. Voronin and V. Khovaylo, Scr. Mater., 2015, 96, 9-12.

40 J. R. Szczech, J. M. Higgins and S. Jin, J. Mater. Chem., 2011, 21, 4037-4055. 